\title{
Fabrication of crystalline Bragg reflectors for high power and integrated optical applications by multi-beam Pulsed Laser Deposition
}

\author{
Katherine A. Sloyan, Timothy C. May-Smith, Michalis Zervas and Robert W. Eason \\ Optoelectronics Research Centre, University of Southampton, Southampton, UK SO17 1BJ \\ kas@orc.soton.ac.uk
}

\begin{abstract}
Tunable crystalline Bragg reflectors for high temperature, high power and integrated optical applications were fabricated via multi-beam Pulsed Laser Deposition. Apodised, $\pi$ phase-shifted and $>99 \%$ reflective quarter-wave structures are presented.

(C) 2011 Optical Society of America
\end{abstract}

OCIS codes: $220.0220,230.1480$.

\section{Introduction}

Bragg gratings are employed in a variety of applications, from sensors to mirrors. While gratings can be fabricated from amorphous materials by a number of methods in both waveguide and planar geometries, their use can be limited in high power or high temperature applications. Crystalline Bragg stacks, however, can be expected to have a much higher resistance to such damage under extreme conditions, as well as having the additional benefit of use in hybrid laser crystals (e.g. integrated mirrors for thin disc lasers). We have developed shuttered multi-beam Pulsed Laser Deposition (PLD) as a method of growing such structures quickly and easily compared to many other techniques, and in geometries that are extremely difficult to grow by other methods, including single-beam PLD. Here we describe a number of sophisticated single-phase crystalline Bragg stacks grown via this technique, including the first example to our knowledge of an apodised crystalline dielectric mirror. Tuning is possible due to a predictable variation in peak wavelength with position on the sample. High temperature and high power laser damage testing is currently underway, with the damage threshold expected to be high in both cases.

\section{Experiment and results}

The shutter technique has been demonstrated previously to fabricate crystalline superlattices [1]. A multi-beam, multitarget PLD setup was combined with computer-controlled shutters to allow growth of distinct layers, by operating shutters alternately, or mixed films with a custom ratio of components, by using shutters to change relative laser repetition rates. The technique allows layered structures to be fabricated much more easily and with many more layers than previously published examples of PLD Bragg growth [2].

Samples described here were composed of $\mathrm{Y}_{3} \mathrm{Al}_{5} \mathrm{O}_{12}$ (YAG) and $\mathrm{Gd}_{3} \mathrm{Ga}_{5} \mathrm{O}_{12}$ (GGG) layers grown on YAG substrates of size $10 \times 10 \times 1 \mathrm{~mm}^{3}$. A common set of deposition conditions was chosen for ease of fabrication and to allow mixed layer growth for grating strength apodisation. Substrates were heated via $\mathrm{CO}_{2}$ laser to temperatures of $\sim 750^{\circ} \mathrm{C}$ to obtain single-phase crystalline growth, confirmed via powder X-ray diffraction (see Figure 1). Optical growth rates per shot were estimated by analysis of spectrophotometry data from single material tester films. As films may not have the same stoichiometry as bulk material, refractive indices were estimated for apodised growth by comparison of simple quarter-wave gratings with a MATLAB model.

Simple quarter-wave Bragg reflectors consisted of alternating layers of thickness $\lambda / 4 n$, where $n$ is the refractive index of the layer. $\pi$ phase-shifted structures were similar with a central layer of thickness $\lambda / 2 n$. The apodised sample consisted of alternating layers of YAG and mixed YAG/GGG, varied in ratios of 9:1, 8:2 and so on to 0:10 and back to 9:1 to obtain an approximately Gaussian grating strength profile. This was achieved by changing the relative repetition rates of the ablating lasers using the shutters. Total deposition time for the thickest structures was around seven hours including chamber pumping and venting; this technique is hence of particular interest for rapid prototyping.

Transmission spectra of a number of representative samples are illustrated in Figure 2. Figure 2a shows the spectrum of a simple quarter-wave Bragg structure with 145 alternating layers and a total film thickness of $\sim 17.6 \mu \mathrm{m}$. A 


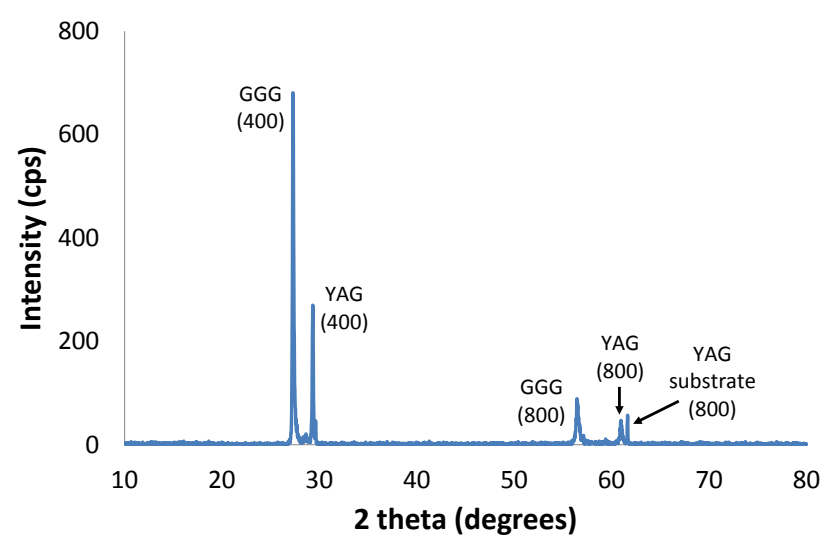

Fig. 1: X-ray diffraction spectrum of a simple Bragg structure showing single-phase crystalline growth

transmission minimum of $<1 \%$ can be observed at a wavelength of $946 \mathrm{~nm}$, corresponding to a peak reflection of $>99 \%$ (absorption is negligible). An increase in transmission of $\sim 50 \%$ at the central wavelength of a $\pi$ phase-shifted structure can be clearly seen in Figure $2 \mathrm{~b}$. This spectrum was obtained using a tuneable Ti:sapphire laser, in order to ensure optimum coherence of the interrogating light. Successful apodising was evinced by the spectra given in Figure 2c: significant reduction of sidebands can be observed relative to a quarter-wave structure.

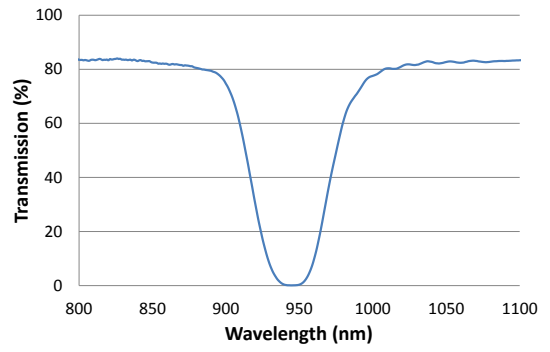

(a) 145 layer simple Bragg structure

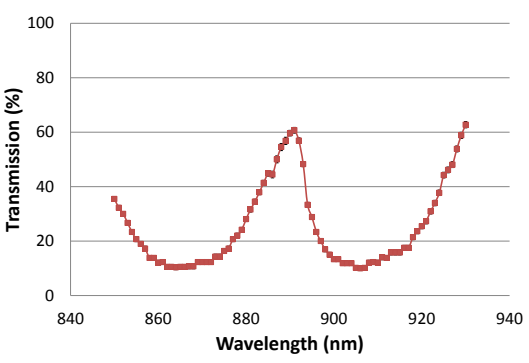

(b) Central peak of a $\pi$ phase-shifted structure

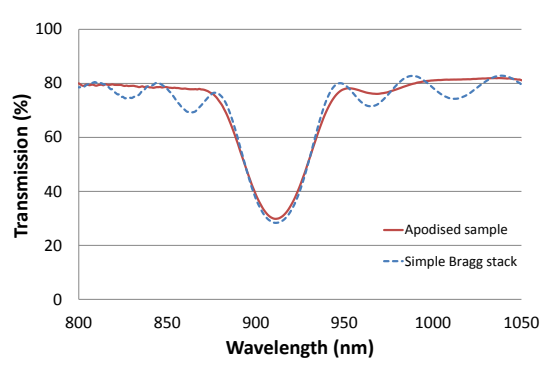

(c) Apodised and simple Bragg spectra

Fig. 2: Transmission spectra of crystalline Bragg gratings obtained via spectrophotometer (Figures 2a and 2c) and Ti:sapphire laser (Figure 2b).

Crystalline Bragg structures have a range of exciting potential applications. Garnet crystals have high melting points (YAG melts at $>1900{ }^{\circ} \mathrm{C}$, for example), good thermal conductivities and high damage thresholds. It is therefore expected that such structures may be suitable for high temperature sensing applications, as well as for rapid production of custom high-power laser mirrors; as such, damage testing is currently under way. In addition, peak wavelength has been tuned by up to $\sim 40 \mathrm{~nm}$ by changing the incidence position on the substrate. This is possible due to film curvature, which is under experimental control. The crystalline nature of the films also raises the possibility of integrated growth, such as the integrated growth of mirrors and laser crystals in thin disc laser structures.

\section{References}

1. K. A. Sloyan, T. C. May-Smith, M. Zervas, R. W. Eason, S. Huband, D. Walker, and P. A. Thomas "Growth of crystalline garnet mixed films, superlattices and multilayers for optical applications via shuttered Combinatorial Pulsed Laser Deposition,” Opt. Express 18, 24679-24687 (2010).

2. J. Sellmann, Ch. Sturm, R. Schmidt-Grund, Ch. Czekalla, J. Lenzner, H. Hochmuth, B. Rheinlnder, M. Lorenz, and M. Grundmann "Structural and optical properties of $\mathrm{ZrO}_{2}$ and $\mathrm{Al}_{2} \mathrm{O}_{3}$ thin films and Bragg reflectors grown by pulsed laser deposition," Phys. Stat. Sol. C. 5, $1240-1243$ (2008). 